\title{
Ajuste oclusal en prótesis completa
}

\author{
Occlusal Adjustment in Complete Dentures
}

Jessica Gabriela Laura Cahuana

(iD) https://orcid.org/0000-0003-3446-1801

\section{Resumen}

El propósito de este reporte de caso fue presentar un procedimiento de ajuste oclusal mediante remontaje clínico previo a la instalación de las prótesis completas en una paciente de sexo femenino de 65 años de edad, en ABEG, LOTEP. El diagnóstico definitivo fue la alteración de la dimensión vertical, edéntulo total superior e inferior. El tratamiento consistió en la confección de prótesis completa superior e inferior por la técnica compleja que incluye el remontaje clínico a partir de la confección de modelos para remontaje, registro de arco facial e intermaxilar y ajuste oclusal en el articulador semiajustable. Este procedimiento es una buena alternativa clínica para lograr una oclusión estable sin lesiones tisulares ni sintomatología dolorosa posteriores a la instalación; además, conserva la salud de las estructuras de soporte.

Palabras clave: ajuste oclusal, dentadura completa

\begin{abstract}
The purpose of this case report was to present an occlusal adjustment procedure through clinical reassembly prior to the installation of complete prostheses in a 65-year-old female patient at ABEG, LOTEP. The definitive diagnosis was the alteration of the vertical dimension, total upper and lower edentulous. The treatment consisted of the fabrication of a complete upper and lower prosthesis using the complex technique that includes clinical reassembly from the fabrication of models for reassembly, facial and intermaxillary arch registration, and occlusal adjustment in the semi-adjustable articulator. This procedure is a good clinical alternative to achieve stable occlusion without tissue injuries or painful symptoms after installation; in addition, it preserves the health of the supporting structures.
\end{abstract}

Keywords: occlusal adjustment, full dentures

\section{Introducción}

Actualmente la confección de prótesis completa convencional es la forma de tratamiento más utilizada para casos de edentulismo total; sin embargo, la determinación del éxito de este tratamiento está ligado a la satisfacción y confort del paciente.1
Durante el proceso del tratamiento pueden surgir errores de origen clínico y laboratorial debido a diversos factores; tales como la contracción volumétrica propia del material durante el acrilizado; además, la fuerza ejercida durante el proceso de enmuflado puede ocasionar movimientos de los dientes protésicos; y, el sobrecalentamiento de la prótesis durante el

Investigador independiente, Tacna, Perú

Maestro en Odontoestomatología 
pulido puede generar alteraciones a nivel oclusal. $^{2-4}$

Varios métodos para la corrección oclusal en prótesis completa son descritos en la literatura; por ejemplo, el ajuste oclusal en boca, el remontaje de laboratorio y el remontaje clínico. Los autores que defienden el ajuste oclusal intraoral indican que las prótesis deben asentarse; para luego realizar el control oclusal intraoral. Sin embargo, esto ocasionaría que estas se desplacen para adecuarse a las interferencias, generando excesiva presión en los tejidos de soporte.$^{5,6} \mathrm{El}$ remontaje de laboratorio, en los inicios, consistía en retirar las prótesis con la precaución de conservar la base del modelo; luego, se realizaba el remontaje en el articulador nuevamente. No obstante, pese a que este proceso se realizaba meticulosamente, surgían discrepancias a nivel oclusal que se corregirian posteriormente en boca. Tradicionalmente, la fabricación de prótesis completa comprende un método estándar y un método complejo, ambos métodos comparten similitudes; sin embargo, tomando en consideración la dificultad clínica del análisis y corrección oclusal intraoral, a causa del desplazamiento de las bases en el soporte mucosa-hueso; ${ }^{8}$ el método complejo involucra la corrección oclusal mediante el procedimiento de remontaje clínico, a partir de la ubicación del eje de bisagra, la transferencia del arco facial, el remontaje después del procesamiento de las prótesis utilizando nuevos registros interoclusales y el ajuste oclusal en céntrica y movimientos excéntricos.,

Al optar por el esquema oclusal balanceado bilateral se espera lograr el mayor número de contactos en céntrica y en movimientos excéntricos. Según la literatura, a diferencia del método estándar de confección de prótesis completas, con el remontaje clínico, se logra mayor número de contactos oclusales con una distribución de baja intensidad más favorable, evidente incluso una semana después de la instalación, ${ }^{10}$ reduciendo considerablemente el número de citas posinstalación, así como la menor incidencia de dolor e inflamación tisular. ${ }^{11,12}$

El propósito de este reporte de caso es presentar un procedimiento de ajuste oclusal mediante remontaje clínico como parte importante del tratamiento de prótesis completas previamente a la instalación de las mismas.

\section{Caso Clínico}

Paciente de sexo femenino de 65 años de edad, en ABEG, LOTEP acude a consulta odontológica, por "necesidad de cambio de prótesis completas deterioradas y desadaptadas". Al examen regional estomatológico, el paciente presentó labios hidratados delgados, presencia de saliva abundante y viscosa. El diagnóstico del paciente fue alteración de la dimensión vertical, edéntulo total superior e inferior. Los objetivos del tratamiento fueron restablecer la dimensión vertical, restablecer la estabilidad oclusal, preservar los tejidos remanentes y restablecer la estética dental. El tratamiento se planificó en dos fases. La primera, la fase correctiva consistió en la confección de prótesis completa superior e inferior y remontaje clínico. La segunda, la fase de mantenimiento consistió en controles a las 24 horas, 7 días, y 21 días.

Después del proceso de acrilizado, se procedió a la eliminación de burbujas o excesos, así como áreas de superficie irregular o bordes afilados. Asimismo, se evaluaron los márgenes de la prótesis en cuanto a grosor y extensión, estos márgenes no deben ser cónicos ni gruesos. También, se procedió a la evaluación de las áreas compresivas por medio de presión digital y oclusal mediante torundas de algodón interpuestas entre las caras oclusales y mediante una pasta indicadora de presión. Luego, se procedió a realizar las pruebas de retención vertical, horizontal y lateral, obtenidas mediante el sellado periférico. Para la correción oclusal, se realizó el procedimiento de remontaje clínico (Figura 1). 


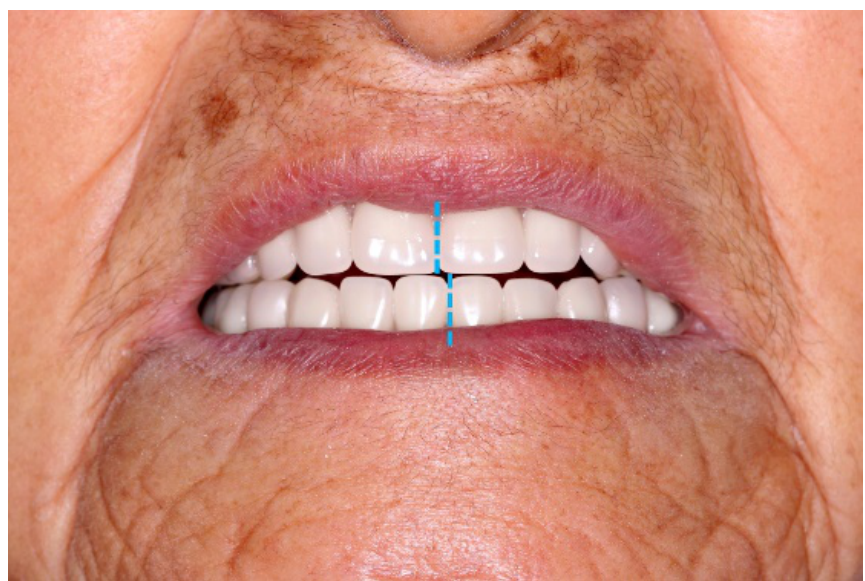

Figura 1. Fotografía inicial de las prótesis acrilizadas, evidenciando puntos prematuros y deslizantes.

Se colocó una mezcla homogenea de silicona de condensacion (Zetalabor) - activador en la parte interna de las prótesis completas acrilizadas, ubicando simultaneamente retenedores de aproximadamente $12 \mathrm{~mm}$ (Figura 2A-D). Se realizó el zocalado en yeso tipo III (Figura 3A y B).

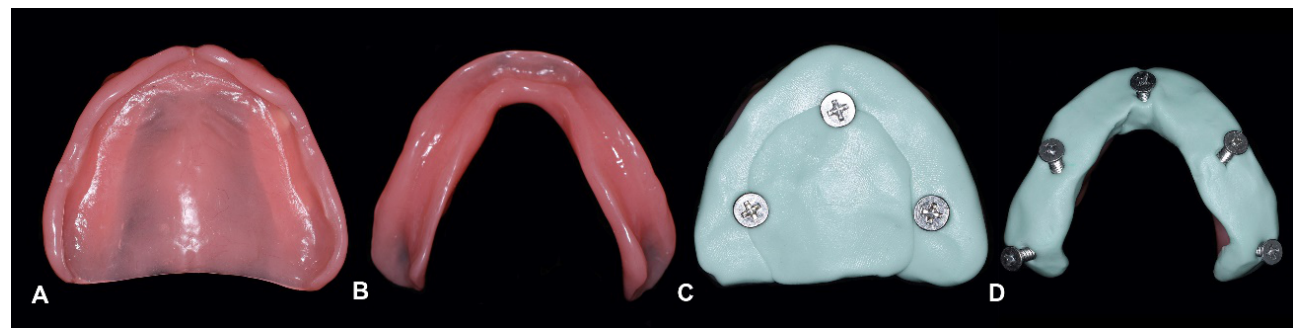

Figura 2. A: Prótesis completa superior. B: Prótesis completa inferior. C: Fabricación de modelo superior para remontaje. D: Fabricación de modelo inferior para remontaje.

Se procedió a la toma de registro de arco facial e intermaxilar en relación céntrica por ser una posición reproducible y repetible (Figura $3 \mathrm{C}$ ). Se llevó al paciente a relación céntrica, se observó la ubicación del primer contacto oclusal, se colocó el material para el registro de la relación maxilo-mandibular (pasta zinquenólica - Cavex Outline) y se pidió al paciente que cierre justo en ese contacto sin ejercer mayor fuerza para evitar que las bases se desplacen.

Se realizó el remontaje en el articulador semiajustable en yeso tipo III (Figura 3D). Se procedió a comparar la posición del primer contacto oclusal en céntrica y se verificó que eran idénticos.

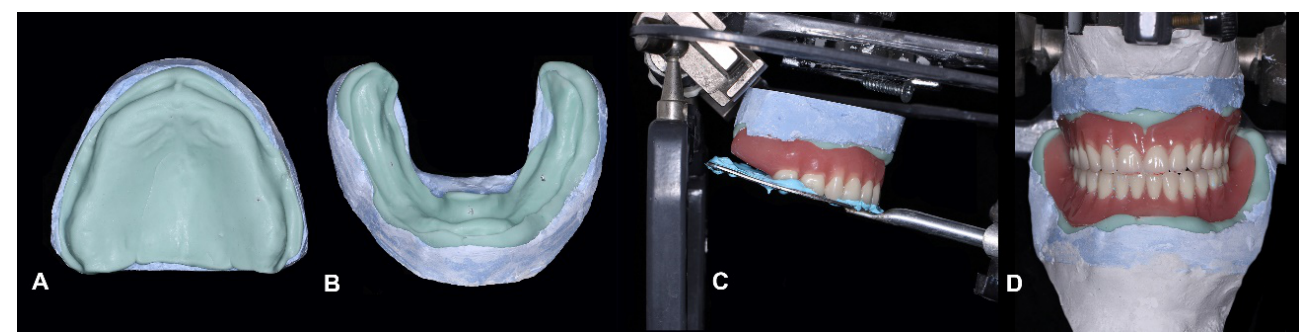

Figura 3. A y B: Modelos para remontaje, zocalados en yeso tipo III. C: Registro de Arco Facial. D: Registro intermaxilar y montaje en articulador. 
La corrección de los contactos oclusales después del remontaje en articulador se realizó con papel de articular de $100 \mu \mathrm{m}$, el cual marca las diferentes fuerzas de presión. Se evidenciaron contactos en relación céntrica insuficientes a nivel de premolares y molares superiores e inferiores (Figura 4A y B). Se procedió al desgaste selectivo preservando las cúspides, las vertientes vestibulares y el esquema oclusal seleccionado hasta lograr contactos en todos los dientes posteriores. Opcionalmente, se puede incluir caninos, sin incluir incisivos, a su vez, se debe mantener el contacto del vástago incisal con la mesa incisal (Figura 4C y D).

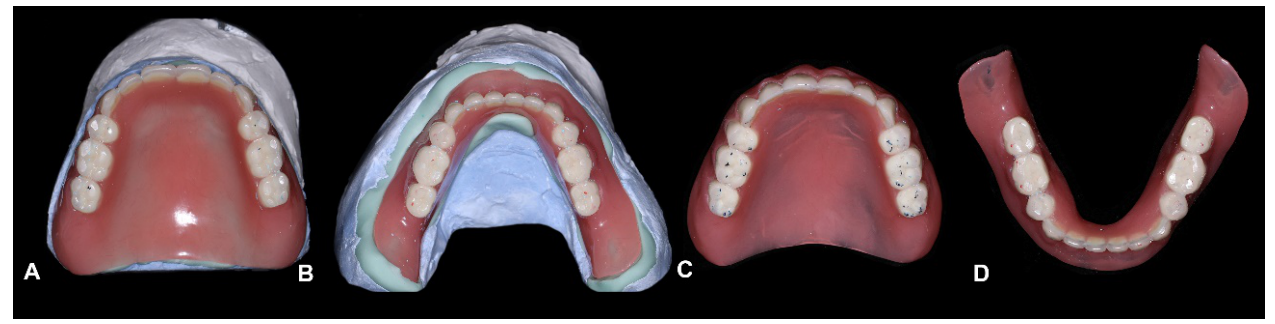

Figura 4. A y B: Primeros contactos en Relación céntrica. C y D: Máximo número de contactos en Relación céntrica.

El siguiente paso fue la eliminación de contactos deflectivos que impedían los contactos posteriores durante el movimiento protrusivo recto; a nivel de incisivos, se lograron contactos simultaneos y uniformes, ya que el overbite en este esquema oclusal es de $0 \mathrm{~mm}$. El desgaste estuvo dirigido hacia vertientes palatinas y linguales debido a razones estéticas. En lo referente al movimiento de lateralidad derecha, la parte superior del articulador se mueve hacia el lado izquierdo por lo que la esfera condilar del lado de trabajo toca la parte posterior de la caja condilar. Es necesario lograr función de grupo en el lado de trabajo con contactos cercanos a las fosas debido a que estos son los lugares para los contactos en la posición retrusiva; a su vez, se debe lograr al menos un contacto en el lado de balance (Figura 5). La misma corrección se realiza en el movimiento de lateralidad izquierda (Figura 5).

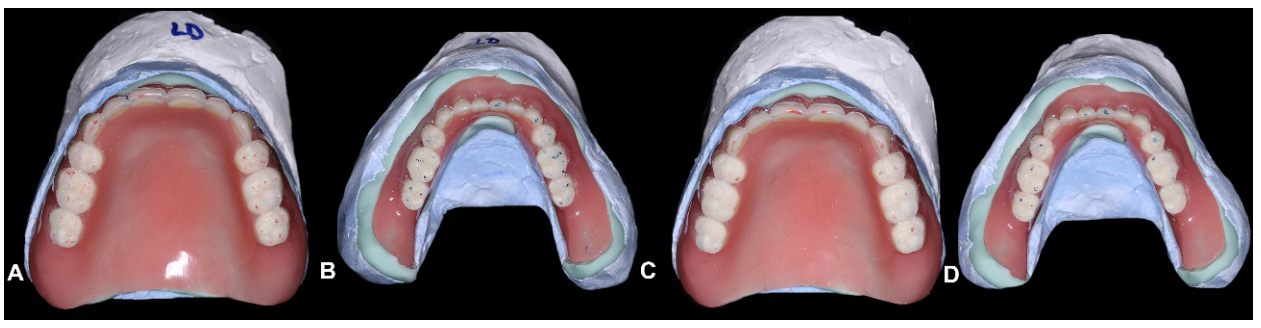

Figura 5. A y B: Contactos logrados en lateralidad derecha. C y D: Contactos logrados en lateralidad izquierda.

En el día de la instalación posterior al remontaje clínico, se procedió a la evaluación oclusal en boca, a fin de confirmar los resultados de la correción oclusal mediante este procedimiento (Figura 6). La paciente regresó después de 24 horas para evaluación, sin manifestar problemas específicos como dolor o incomodidad. Posteriormente, se realizaron controles a los 7 días y 21 días, en ellos se observó el mantenimiento del esquema oclusal establecido y ausencia de sintomatología dolorosa. 


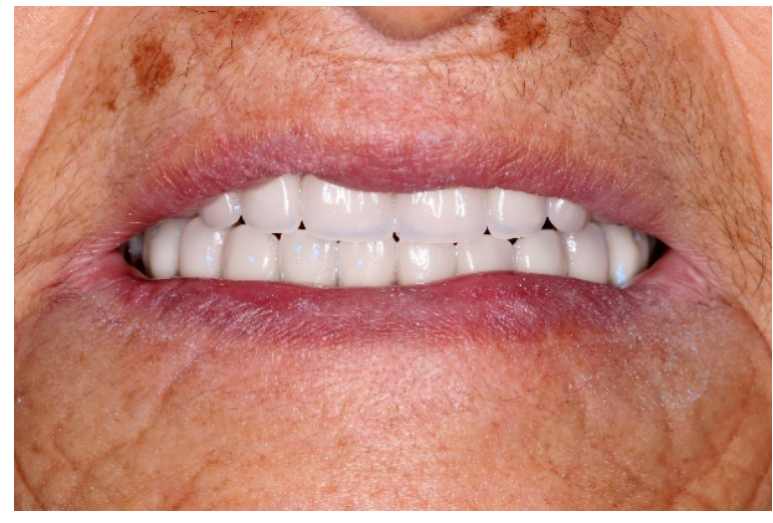

Figura 6. Fotografía posterior al control oclusal por medio del remontaje clínico.

\section{Discusión}

Shigli et al., ${ }^{(13)}$ en un estudio clínico controlado aleatoriamente, indican que la combinación de los procedimientos de remontaje clínico y laboratorial junto a las correcciones oclusales reducen significativamente el número de visitas posinstalación, la presencia de dolor durante la masticación y la incidencia de irritación tisular en comparación a los pacientes en los que no se realizan uno o ambos procedimientos.

Existen factores físicos, biológicos y protésicos que determinan la calidad funcional de las prótesis completas. Primero, respecto a los factores físicos, podemos decir que el contacto entre la base de la prótesis, la mucosa y la saliva permiten la retención por adhesión, cohesión y presión atmosférica. Segundo, los factores biológicos dependen del equilibrio neuromuscular y del contenido y la cantidad de saliva; en este punto, el reborde residual puede servir de retención hasta cierto punto, así como la mucosa móvil y los músculos pueden favorecer la estabilización. Tercero, los factores protésicos incluyen la disposición y los contactos oclusales de los dientes de la prótesis. Por lo tanto, el control y la corrección de los contactos oclusales durante la confección de la prótesis, la polimerización de las bases de la prótesis y el cuidado posterior del paciente son muy importantes. ${ }^{14}$

Asimismo, la oclusión varía constantemente dependiendo del tono muscular, la remodelación de las estructuras de soporte, el desgaste dentario y la salud mental. ${ }^{15}$ El remontaje puede ayudar a reducir y prevenir los cambios oclusales y mejorar el confort. ${ }^{16}$ Este procedimiento puede repetirse varias veces, después de las 2 a 6 semanas posteriores a la instalación, siendo recomendable realizar el control oclusal al año como mínimo.

Finalmente, se ha descrito la implicancia clínica de este procedimiento sobre la actividad de los músculos masticatorios y el confort de los pacientes portadores de prótesis, ya que los resultados muestran que este procedimiento clínico, aumenta significativamente el rendimiento masticatorio y la actividad electromiográfica, preserva los tejidos blandos y reduce el número de ajustes posteriores a la instalación, ${ }^{17}$ permitiendo realizar un control completo de la oclusión, eliminando las variables de desplazamiento del tejido o movimiento de las bases de la prótesis. ${ }^{12}$

\section{Conclusión}

Se concluyó que, el ajuste oclusal mediante remontaje clínico es una buena alternativa clínica para lograr una oclusión estable, sin lesiones tisulares ni sintomatología dolorosa posterior a la instalación, además de conservar la salud de las estructuras de soporte.

\section{Referencias}

1. Verhaeghe TV, Linke BA, Cable CE, Mostafa N. Clinical remounting of complete dentures: A systematic review. J Prosthet Dent. Apr 2019;121(4):60410 . D 0 i : 10.1016/j.prosdent.2018.06.017

2. Badel T, Pandurić J, Kraljevic Simunkovic S, Dulcic N. Checking the occlusal relationships of complete dentures via a remount procedure. Int J Periodontics Restorative Dent. Apr 2007; 27(2):181-92. PMID: 17514890.

3. Celebic A, Knezovic-Zlataric D, Papic M, Carek V, Baucic I, Stipetic J. Factors related to patient satisfaction with complete denture therapy. J Gerontol A Biol Sci Med Sci. Oct 2003; $58(10)$ : M $948-53$. D o i : 10.1093/gerona/58.10.m948.

4. Ghani F, Kikuchi M, Lynch C, Watanabe $M$. Effect of some curing methods on 
acrylic maxillary denture base fit. Eur $\mathrm{J}$ Prosthodont Restor Dent. Sept 2010;18(3):132-8. PMID: 21077423.

5. Saponaro PC, Yilmaz B, Heshmati RH, McGlumphy EA. Clinical performance of CAD-CAM-fabricated complete dentures: A cross-sectional study. J Prosthet Dent. Sept 2016; 116(3):4315.Doi: 10.1016/j.prosdent.2016.03.017.

6. Samant A, McDermott I, Cinotti WR. Delivery complete dentures: preventing problems after insertion. Gen Dent. May-jun 1984; 32(3):229-31.PMID: 6394428.

7. Beresin VE, Schiesser FJ. The neutral zone in complete dentures. J Prosthet Dent. Oct 1976; 36(4):356-67. Doi: 10.1016/0022-3913(76)90155-4. PMID: 787504.

8. Savabi G, Savabi O, Dastgheib B, Nejatidanesh F. Effect of the processing cycle on dimensional changes of heatpolymerized denture base resins. Dent Res J (Isfahan). Jul-agt 2015; 12(4):301-6. Doi: 10.4103/17353327.161423. PMID: 26288618.

9. Hickey JC, Henderson D, Straus R. Patient response to variations in denture technique. I. Design of a study. J Prosthet Dent. Agto 1969; 22(2):158-70. Doi: 10.1016/0022-3913(69)90243-1.

10. Polyzois $G$, Karkazis $H$, Zissis A. Remounting procedures for complete dentures: a study of occlusal contacts by the photocclusion technique. Quintessence Int. Oct 1991; 22(10):8115. ISSN 1936-7163
11. Al-Quran FA. A clinical evaluation of the clinical remount procedure. J Contemp Dent Pract. Feb 2005; 6(1):48-55. PMID: 15719076.

12. Nimmo A. Clinical remount for complete dentures. Quintessence Int. Apr 1988; 19(4):273-7. PMID: 3077674.

13. Shigli K, Angadi GS, Hegde P. The effect of remount procedures on patient comfort for complete denture treatment. J Prosthet Dent. Ene 2008; 99(1):66-72. Doi: 10.1016/S0022-3913(08)60011-6.

14. Badel T, Panduric J, Kraljevic S, Dulcic $\mathrm{N}$. Checking the occlusal relationships of complete dentures via a remount procedure. Int J Periodontics Restorative Dent. Apr 2007; 27(2):18192. PMID: 17514890.

15. Berry DC, Singh BP. Daily variations in occlusal contacts. J Prosthet Dent. Sept $1983 ; 50(3): 386-91$. Do i : 10.1016/s0022-3913(83)80099-7.

16. Firtell DN, Finzen FC, Holmes JB. The effect of clinical remount procedures on the comfort and success of complete dentures. J Prosthet Dent. Ene 1987; 57(1):53-7. Doi: 10.1016/00223913(87)90116-8

17. Mostafa MMM, Ezzat AE, Awad EA, Amine KKE, Amine HKE. Effect of Clinical Remount Procedures on Masticatory Muscle Activity and Comfort of Complete Denture Wearers. Curr Sci Int. 2019;8(01):35-41. ISSN: 20774435 . D i s p o n i ble e n : http://www.curresweb.com/csi/csi/2019 /35-41.pdf
Correspondencia:

cdgabrielaura@hotmail.com
Fecha de recepción : 26 de julio de 2020

Fecha de aceptación : 02 de septiembre de 2020 